\title{
Gallbladder duplication in a child - case report
}

\author{
Katarzyna Zdanowicz', Monika Kowalczuk-Krystoń1, Małgorzata Wojtkowska², \\ Beata Zalewska-Szajda², Dariusz M. Lebensztejn' \\ 'Department of Paediatrics, Gastroenterology, Hepatology, Nutrition, and Allergology, Medical University of Bialystok, \\ Bialystok, Poland \\ 2Department of Imaging Diagnostics, University Clinical Hospital in Bialystok, Bialystok, Poland
}

\section{ABSTRACT}

Congenital malformations of the gallbladder are rare and may coexist with other abnormalities of the biliary or vascular tree. One such abnormality is duplication of the gallbladder that can be asymptomatic or can present as typical gallbladder pathologies like cholelithiasis, cholecystitis, cholangitis, or pancreatitis. Imaging diagnosis is essential in identifying anatomical abnormalities of the gallbladder. The aim of the presentation is to describe the case of a 16-year-old girl admitted to hospital with abdominal pain. Abdomen ultrasound and magnetic resonance cholangiopancreatography detected a duplicated gallbladder. In the patient other clinical, laboratory, and radiological features of the gallbladder or bile duct pathology were not observed. In the case of this congenital anomaly, if it occurs asymptomatically, conservative and surgical treatment is not indicated.

\section{KEY WORDS:}

children, congenital abnormalities, duplicated gallbladder.

\section{INTRODUCTION}

Several gallbladder congenital abnormalities have been reported, which may be related to the number, shape, and position of the organ [1]. One of them is gallbladder duplication, with an incidence one per 4000 births [2]. This congenital anomaly is often asymptomatic but may be associated with pathologies like cholelithiasis, cholecystitis, cholangitis, and pancreatitis [3]. In addition, gallbladder duplication requires special attention during cholecystectomy because it is relevant to iatrogenic bile duct injuries during surgery [4].

\section{CASE REPORT}

A 16-year-old girl was admitted to the hospital with a one-month history of abdominal pain, nausea, and vomiting. Emesis was not associated with fever or diarrhoea. The medical history of the patient was remarkable for Helicobacter pylori eradication two years earlier. Her general condition was good. Physical examination revealed pain of the upper abdomen. Laboratory data showed a normal white blood cell count (5900 cells/ul) and serum C-reactive protein level $(0.3 \mathrm{mg} / \mathrm{l})$. Serum total bilirubin $(0.7 \mathrm{mg} / \mathrm{dl})$, alanine aminotransferase (7 U/l), $\gamma$-glutamyltransferase (12 U/l), amylase (36 U/l), and lipase (15 U/1) levels were within normal limits. Due to a history of $H$. pylori infection and epigastric abdominal pain, esophagogastroduodenoscopy was performed and showed antrum erythema and granular changes; rapid urease test was negative. Pathologic examination of gastric biopsy specimens revealed chronic gastritis with single erosion, $H$. pylori infection was not observed. In treatment a proton pump inhib-

\section{ADDRESS FOR CORRESPONDENCE:}

Katarzyna Zdanowicz, Department of Paediatrics, Gastroenterology, Hepatology, Nutrition, and Allergology, Medical University of Bialystok, 17 Waszyngtona St., 15-274 Bialystok, Poland, ORCID: 0000-0000-0002-0755-911X, e-mail: kazdanowicz@gmail.com 


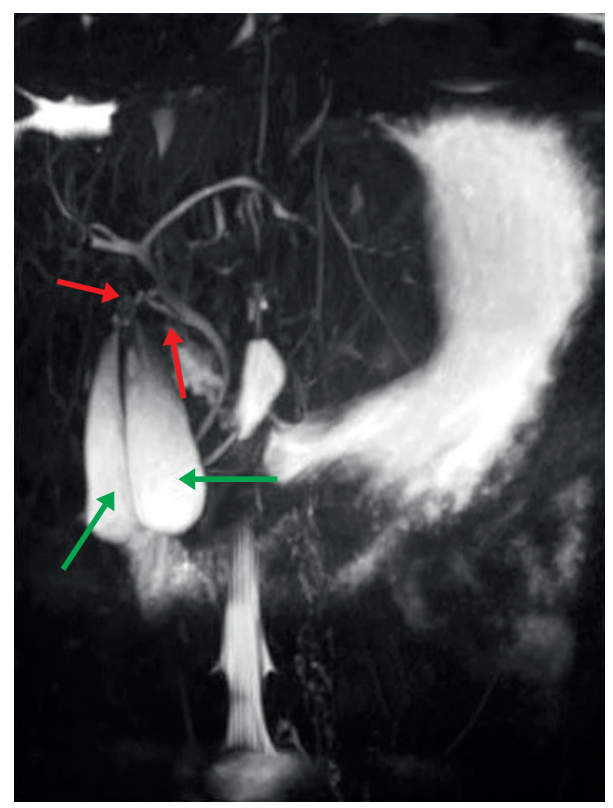

FIGURE 1. Magnetic resonance cholangiopancreatography of two separate gallbladders (green arrows) and two cystic ducts (red arrows)

itor (omeprazole) was used with a complete clinical response. An abdominal ultrasound showed two cystic structures with normal gallbladder wall thickness and no evidence of stones, with a small amount of echogenic sludge within one of them. There was no evidence of intra- or extrahepatic biliary tree dilatation. In magnetic resonance cholangiopancreatography imaging (MRCP), two separate gallbladders and two cystic ducts were visualised without dilatation of the common bile duct and no intrahepatic biliary dilatation (Figs. 1 and 2). Due to the lack of gallbladder disease symptoms, treatment was not carried out. After this management, the patient underwent a full recovery. On follow-up controls the girl is doing well, and she is still being observed in a Gastroenterology Outpatient Clinic.

\section{DISCUSSION}

Duplicated gallbladder is a rare congenital malformation with equal occurrences in females and males [1]. In the beginning of the fourth week of gestation hepatic diverticulum becomes the anlage for the development of the liver, extrahepatic biliary ducts, gallbladder, and ventral pancreas. The cystic duct and gallbladder appear from the caudal bud [5]. An exuberant budding during hepatic diverticulum division is the probable cause of malformation [6]. The first described duplicated gallbladder was noticed in ancient times - 35 BC [7]. Duplication of gallbladder is classified according to anatomic variants. The most widely accepted classifications are:

- vesica fellea divisa (bilobed or bifid gallbladder, double gallbladder with a common neck),

- vesica fellea duplex (double gallbladder with two cystic ducts) which includes Y-shaped type (the two cystic ducts uniting before entering the common bile duct)

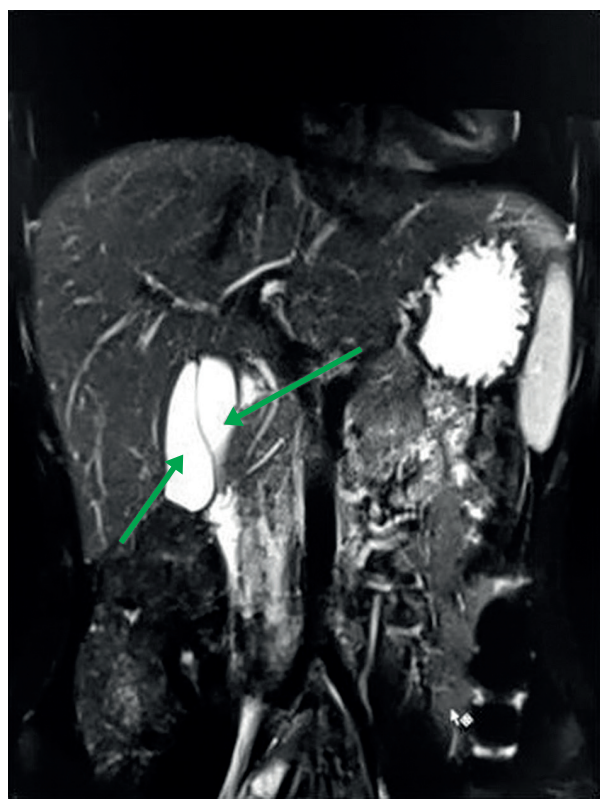

FIGURE 2. Magnetic resonance cholangiopancreatography of two separate gallbladders (green arrows)

and $\mathrm{H}$-shaped type (ductular type, the two cystic ducts entering separately into the biliary tree) $[2,8]$.

The incidence of cases from the first group is about $45.1 \%$ while the second group is $54.9 \%$. $\mathrm{H}$-shaped type is the most common, which accounts for about $47.2 \%$ [9]. Combinations of gallbladder and hepatic artery malformations are observed, which give valuable information for surgeons [7]. No specific clinical significance associated with gallbladder duplication has been reported, but patients may have health problems caused by the occurrence of cholelithiasis, cholecystitis, cholangitis, empyema, torsion, cholecystocolic fistula, and pancreatitis [3, 8]. Symptoms connected with a duplicated gallbladder are similar to those observed in a single organ. Differential diagnosis includes gallbladder diverticula, gallbladder fold, choledochal cyst, pericholecystic fluid, focal adenomyomatosis, and intraperitoneal fibrous bands [4]. The first-choice method of screening for patients with suspected biliary disorder in ultrasound (US). At US, assessment of wall thickness, lumen pathology, and the number of the gallbladders is used, but the biliary tree is usually not identified, so it is impossible to distinguish true duplication from bilobed gallbladder [10]. MRCP is a further evaluation in the diagnosis of biliary pathology [11]. Helical CT scan may also be useful [12]. Due to the occurrence of less invasive radiology tests, oral cholecystography, scintigraphy, and percutaneous transhepatic cholangiography are not commonly used [11].

As mentioned, this congenital anomaly is often asymptomatic. If complications occur, the treatment is similar to that of single gallbladder. If one or both gallbladders are the cause of symptoms, (open or laparoscopic) cholecystectomy should be done for both gallbladders [3]. Preventive removal of two asymptomatic gallbladders is not recommended. No evidence was found for an 
increased risk of duplex gallbladder pathology [13]. In our patient after ultrasound examination a gallbladder duplication was detected, and it was confirmed by MRCP. No abnormalities were observed in blood laboratory tests. Based on a literature review, most cases of duplicated gallbladder are identified in adulthood. Duplicate gallbladder with duodenal duplication cyst in a four-year-old boy was described [14]. We did not observe the simultaneous occurrence of both abnormalities in our patient. In addition, only six cases of gallbladder duplication have been reported in the perinatal period [15]. To date, individual cases of triple gallbladders have been reported [16]. One of the most interesting is a case report of triple gallbladder with cholecystitis and cholelithiasis in one gallbladder, papillary adenocarcinoma in the second gallbladder, and a disease-free intrahepatically located third gallbladder [17]. A wide range of disorders may affect the gallbladder, and duplication of the gallbladder, even if it is rare, is one that requires special attention.

\section{DISCLOSURE}

The authors declare no conflict of interest.

\section{REFERENCES}

1. Praseedom RK, Mohammed R. Two cases of gall bladder agenesis and review of the literature. Hepatogastroenterology 1998; 5: 954-955.

2. Boyden EA. The accessory gallbladder: an embryological and comparative study of aberrant billary vesicles occurring in man and the domestic mammals. Am J Anat 1926; 38: 177-231.

3. Romero EXA, Salazar PFG, Chandi JAE, et al. Gallbladder duplication and cholecystitis. J Surg Case Rep 2018; 7: 1-3.

4. Pillay Y. Gallbladder duplication. Int J Surg Case Rep 2015; 11: 18-20.

5. Ando H. Embryology of the Biliary. Tract. Dig Surg 2010; 27: 87-89.

6. Lamah M, Karanjia ND, Dickson GH. Anatomical variations of the extrahepatic biliary tree: review of the world literature. Clin Anat 2001; 14: 167-172.

7. Udelsman R, Sugarbaker PH. Congenital duplication of the gallbladder associated with an anomalous right hepatic artery. Am J Surg 1985; 149: 812-815.

8. Desolneux G, Mucci S, Lebigot J, et al. Duplication of the Gallbladder. A Case Report. Gastroenterol Res Pract 2009; 2009: 483473.

9. Paraskevas GK, Raikos A, Ioannidis O, Papaziogas B. Duplicated gallbladder: surgical application and review of the literature. IJAE 2011; 116: 61-66.

10. Gocman R, Yesilkaya Y. Imaging findings of gallbladder duplication due to two cases: case report and review of literature. J Ultrasound Med 2012; 14: 358-360.

11. Mazziotti S, Minutoli F, Blandino A, et al. Gallbladder duplication: MR cholangiography demonstration. Abdom Imaging 2001; 26: 287-289.

12. Ozgen A, Akata D, Arat A, et al. Gallbladder duplication: Imaging findings and differential considerations. Abdom Imaging 1999; 24: 285-288.

13. Goh YM, Goh YL, Ewan LC, et al. A case report of duplex gallbladder and review of the literature. Int J Surg Case Rep 2015; 14: 179-181.
14. Menon P, Rao KL, Thapa BR, et al. Duplicated gall bladder with duodenal duplication cyst. J Pediatr Surg 2013; 48: e25-28.

15. Maggi U, Farris G, Carnevali A, et al. Prenatal and accurate perinatal diagnosis of type $2 \mathrm{H}$ or ductular duplicate gallbladder. BMC Pediatr 2018; 18: 38.

16. Alicioglu B. An incidental case of triple gallbladder. World J Gastroentrol 2007; 13: 2004-2006.

17. Roeder WJ, Mersheimer WL, Kazarian KK. Triplication of the gallbladder with cholecystitis, cholelithiasis, and papillary adenocarcinoma. Am J Surg 1971; 121: 746-748. 